\title{
Pediatric Pseudotumor Cerebri Syndrome: Diagnostic Criteria and Classification
}

\author{
Claire A. Sheldon ${ }^{1}$ Grace L. Paley ${ }^{1} \quad$ Shannon Beres ${ }^{1}$ \\ ${ }^{1}$ Division of Ophthalmology, Children's Hospital of Philadelphia, \\ Philadelphia, Pennsylvania, United States \\ 2 Division of Endocrinology, Children's Hospital of Philadelphia, \\ Philadelphia, Pennsylvania, United States \\ J Pediatr Neurol 2015;13:8-10.
}

Shana E. McCormack ${ }^{2}$ Grant T. Liu ${ }^{1}$

\begin{abstract}
Address for correspondence Grant T. Liu, MD, Division of Ophthalmology, Children's Hospital of Philadelphia, Wood Pavilion, 3401 Civic Center Blvd, Philadelphia, PA 19104, United States (e-mail: liug1@email.chop.edu).
\end{abstract}

\author{
Abstract \\ Keywords \\ - idiopathic intracranial \\ hypertension \\ - pseudotumor cerebri \\ syndrome \\ - children \\ - diagnostic criteria \\ - classification
}

Pseudotumor cerebri syndrome (PTCS) is defined by the presence of elevated intracranial pressure in the context of normal brain parenchyma and normal cytologic and chemical analyses of the cerebrospinal fluid. The presenting signs and symptoms of PTCS are various and usually include headache, papilledema, and visual disturbances (e.g., vision loss, double vision). Idiopathic intracranial hypertension, which is the primary form of PTCS, generally affects postpubertal overweight women of childbearing age. Secondary PTCS refers to a clinical diagnosis of PTCS attributable to one or more of a variety of identifiable causes, including venous sinus thrombosis, medications, and medical conditions other than obesity alone. The diverse clinical spectrum of pediatric PTCS is becoming increasingly recognized and recent research in this field is aiming to identify a common pathophysiology. We hereby review the recent diagnostic criteria and classification of pediatric PTCS.

\section{Introduction}

Patients with pseudotumor cerebri syndrome (PTCS) have elevated intracranial pressure (ICP) of unclear etiology. This diagnosis of exclusion requires the presence of normal brain parenchyma on neuroimaging and normal cerebrospinal fluid (CSF) composition without evidence of infection or neoplasm. The diagnosis of PTCS was initially based on the modified Dandy criteria. ${ }^{1,2}$ Subsequent modifications have been made and accepted, based on increasing recognition of typical signs and symptoms of this disorder. ${ }^{3,4}$ The most recent updated diagnostic criteria for PTCS in adults and children were published in $2013^{3}$ and incorporated timely insights into common neuroimaging characteristics of raised ICP and reference ranges of normal CSF opening pressure.

\section{Diagnostic Criteria for Pediatric PTCS}

The recently updated diagnostic criteria for pediatric PTCS ${ }^{3}$ are illustrated in - Fig. 1. The accurate diagnosis of PTCS is

received

January 25, 2015 accepted after revision January 30, 2015
Issue Theme Pediatric Pseudotumor Cerebri Syndrome; Guest Editors: Vincenzo Salpietro, MD, Martino Ruggieri, PhD, and Conrad E. Johanson, PhD first based on the presence or absence of papilledema. In contrast to optic disc edema, papilledema is a term specifically reserved for optic nerve head swelling because of raised ICP. A careful dilated fundus examination is typically sufficient to identify papilledema; however, orbital ultrasound, including a 30-degree test by an experienced ultrasonographer, may be a useful adjunctive test. In the presence of papilledema or if clinical suspicion remains high in the absence of papilledema, the diagnosis of PTCS requires the presence of normal brain parenchyma on contrast-enhanced neuroimaging, such as magnetic resonance imaging (MRI) or computed tomography (CT). In patients with clinical characteristics typical of most PTCS cases (e.g., postpubertal, female, and obese; see the following discussion), an MRI venography is not routinely necessary, but should be considered for those patients at high risk for cerebral venous sinus thrombosis. Venous imaging is required for all atypical patients. Next, a lumbar puncture is necessary to document elevated ICP. Most often, opening pressure greater than

Copyright $\odot 2015$ Georg Thieme Verlag KG, Stuttgart · New York

DOI http://dx.doi.org/ 10.1055/s-0035-1555145. ISSN 1304-2580. 


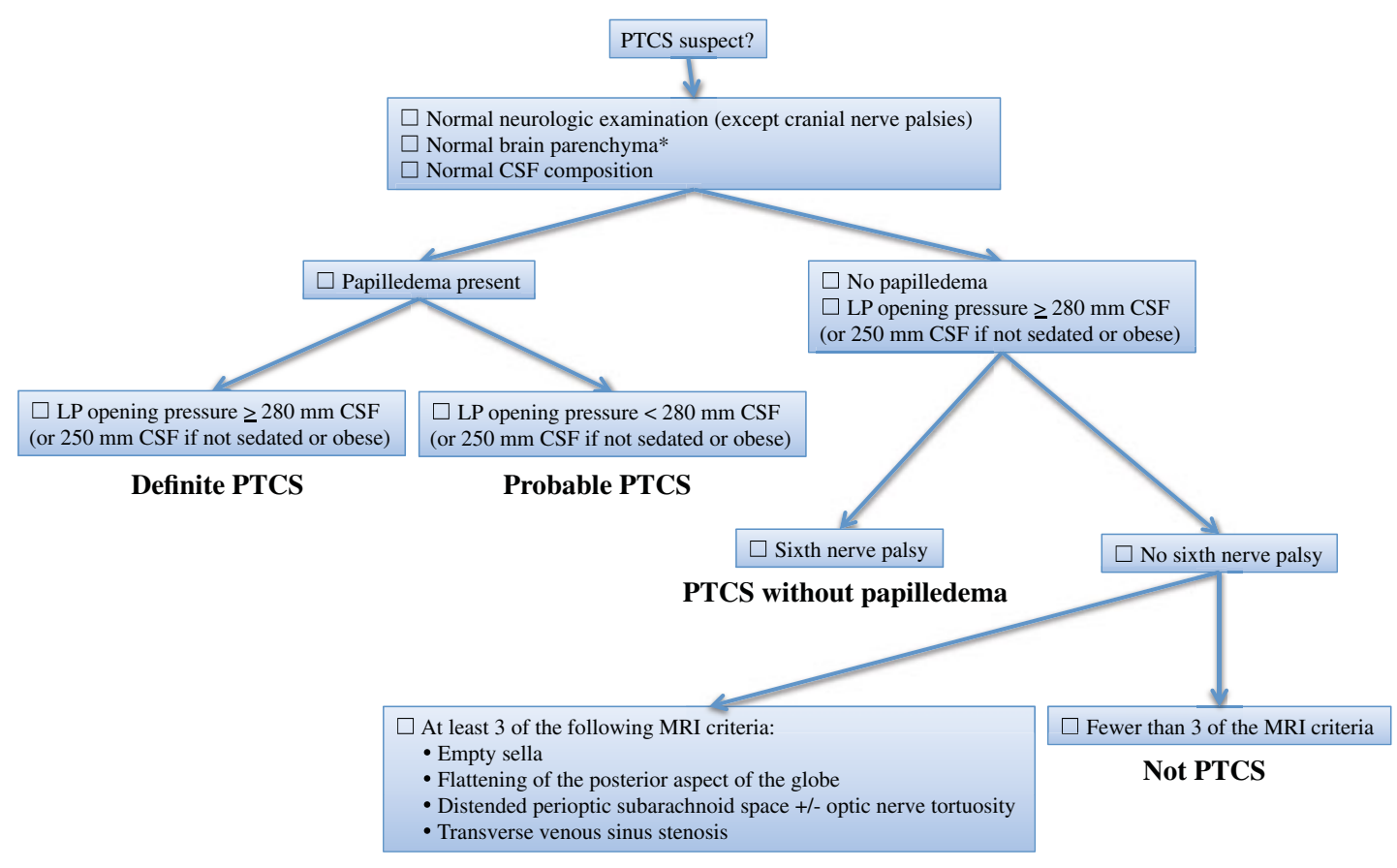

Suggested PTCS

*As seen on contrast-enhanced MRI neuro-imaging for typical patients (female and obese), plus MR-venography for atypical patients (male or nonobese female); or contrast-enhanced CT if MRI is not obtainable.

Fig. 1 Flowchart based on the recent diagnostic criteria for PTCS. ${ }^{3}$ CSF, cerebrospinal fluid; CT, computed tomography; LP, lumbar puncture; MRI, magnetic resonance imaging; PTCS, pseudotumor cerebri syndrome.

$280 \mathrm{mmH}_{2} \mathrm{O}$ in children is considered elevated; although greater than $250 \mathrm{mmH}_{2} \mathrm{O}$ is considered elevated in those not sedated during the lumbar puncture and nonobese children. ${ }^{5}$ CSF composition should be normal, with necessary CSF analyses including cell count, cytology, and concentrations of glucose and protein.

Thus, in the presence of papilledema, key diagnostic requirements include (1) normal brain parenchyma on neuroimaging with contrast-enhanced MRI or CT, (2) normal venous imaging with MRI- or CT-venography in select cases, and (3) normal CSF composition on lumbar puncture. As outlined in recently revised diagnostic criteria for PTCS, ${ }^{6}$ with elevated ICP, a diagnosis of definite PTCS can be made; if a normal ICP is measured, a diagnosis of probable PTCS can be made (-Fig. 1 ).

In the absence of papilledema, key diagnostic requirements include (1) normal brain parenchyma on neuroimaging with contrast-enhanced MRI or CT and (2) elevated ICP and normal CSF composition on lumbar puncture. With these features, in the added presence of unilateral or bilateral sixth nerve palsy, the diagnosis of definite PTCS can be made ${ }^{3}$; however, without a sixth nerve palsy, the diagnosis of suggested PTCS can be made if key neuroimaging features are observed (-Fig. 1). These neuroimaging features include distension of the perioptic subarachnoid space with or without optic nerve tortuosity, flattening of the posterior aspect of the globe, an empty sella, and transverse venous sinus stenosis. ${ }^{3}$ As a group, the required neuroimaging features are highly suggestive of PTCS. ${ }^{6}$ Symptom changes after a lumbar puncture and headache characteristics are not diagnostic of PTCS under the revised criteria.

\section{Classification of Pediatric PTCS}

PTCS is classified as either primary or secondary ( - Table $\mathbf{1}$ ). Primary PTCS is also referred to as idiopathic intracranial hypertension. ${ }^{3}$ In the adult population, the typical patient with primary PTCS is a female of reproductive age who is overweight and/or has a history of recent weight gain. Primary PTCS is certainly seen in the pediatric population, although its epidemiology is complex and characterized by a multifaceted relationship between obesity, pubertal status, and sex. Adolescents with PTCS are more frequently obese and female, suggesting that the risk factors for developing PTCS in this age group may be similar to those in adults. ${ }^{7}$ On the other hand, young, prepubertal children are less likely to be obese, are equally male and female, and may present without symptoms of headache or visual blurring. ${ }^{8,9}$ Ongoing studies are examining the relative influence of sex, age, and key pubertal stages on the epidemiology of pediatric PTCS. ${ }^{10}$ These observations highlight the need for vigilance by the medical provider, not only for overweight and obese children but also for young, thin children who may present with atypical or no symptoms.

Secondary PTCS refers to a clinical diagnosis of PTCS attributable to one or more of a variety of identifiable causes, including venous sinus thrombosis, medications, and medical conditions other than obesity alone. Some of the most 
10 Diagnostic Criteria in Pseudotumor Cerebri Syndrome Sheldon et al.

Table 1 Classification of pseudotumor cerebri syndrome ${ }^{3}$

\begin{tabular}{|l|}
\hline Classification \\
\hline Primary pseudotumor cerebri \\
\hline Idiopathic intracranial hypertension \\
\hline Secondary pseudotumor cerebri (most common causes) \\
\hline Cerebral venous abnormalities: sinus venous thrombosis \\
\hline Medications: tetracycline derivatives, vitamin A and retinoids, lithium \\
\hline Hormones: anabolic steroids, withdrawal of chronic corticosteroid use, human growth hormone, progestin \\
\hline Medical conditions: Addison disease, hypoparathyroidism, anemia (severe), renal failure, Down syndrome, Turner syndrome \\
\hline
\end{tabular}

common causes of secondary PTCS include withdrawal from chronic corticosteroids, marked anemia, exposure to tetracycline-related antibiotics, and synthetic growth hormone or vitamin A derivative medications. ${ }^{3,4,11}$ Together, these cases appear to lack a unifying pathophysiologic mechanism; however, further research into the pathophysiology of PTCS may identify common themes. $^{12}$

In conclusion, children with PTCS have elevated ICP of unclear etiology. This diagnosis requires the presence of normal brain parenchyma on neuroimaging and normal CSF composition without evidence of infection or neoplasm. PTCS can be a highly morbid complication of obesity; however, the diverse clinical spectrum of pediatric PTCS is becoming increasingly recognized. Accurate diagnosis follows clear guidelines, based on clinical examination, neuroimaging, and CSF analysis.

\section{References}

1 Dandy WE. Intracranial pressure without brain tumor: diagnosis and treatment. Ann Surg 1937;106(4):492-513

2 Smith JL. Pseudotumor cerebri. Trans Am Acad Ophthalmol Otolaryngol 1958;62(3):432-440

3 Friedman DI, Liu GT, Digre KB. Revised diagnostic criteria for the pseudotumor cerebri syndrome in adults and children. Neurology 2013;81(13):1159-1165
4 Friedman DI, Jacobson DM. Diagnostic criteria for idiopathic intracranial hypertension. Neurology 2002;59(10):1492-1495

5 Avery RA, Shah SS, Licht DJ, et al. Reference range for cerebrospinal fluid opening pressure in children. N Engl J Med 2010;363(9): 891-893

6 Mallery R, Woo J, Tamhankar M, et al. MRI findings of elevated intracranial pressure in pseudotumor cerebri syndrome with and without papilledema [abstract]. Neurology 2014;82(10):S39.006

7 Brara SM, Koebnick C, Porter AH, Langer-Gould A. Pediatric idiopathic intracranial hypertension and extreme childhood obesity. J Pediatr 2012;161(4):602-607

8 Cinciripini GS, Donahue S, Borchert MS. Idiopathic intracranial hypertension in prepubertal pediatric patients: characteristics, treatment, and outcome. Am J Ophthalmol 1999;127(2):178-182

9 Balcer LJ, Liu GT, Forman S, et al. Idiopathic intracranial hypertension: relation of age and obesity in children. Neurology 1999; 52(4):870-872

10 Sheldon CA, Lin GL, Burrows EK, Chilluti MR, McCormack SE, Liu GT. Pediatric primary pseudotumor cerebri syndrome (PTCS): new insights from detailed endocrine assessments [abstract]. San Juan, Puerto Rico. North American Neuro-Ophthalmology Society. 40th Annual Meeting; 2014

11 Paley GL, Sheldon CA, Burrows EK, Chilutti MR, Liu GT, McCormack SE. Overweight and obesity in pediatric secondary pseudotumor cerebri syndrome. Am J Ophthalmol 2015;159(2):344-52.e1

12 Sheldon CA, Kwon YJ, Liu GT, McCormack SE. An integrated mechanism of pediatric pseudotumor cerebri syndrome: evidence of bioenergetic and hormonal regulation of cerebrospinal fluid dynamics. Pediatr Res 2015;77(2):282-289 